\title{
Development of an Engineering Portfolio System
}

\author{
Warren Stiver, Jerry An, Ben Millen, Ryan Connors, Katherine Sorensen \\ School of Engineering, University of Guelph, Guelph, ON, Canada N1G 2W1 \\ wstiver@uoguelph.ca
}

\begin{abstract}
An electronic engineering portfolio system is under development. This paper briefly describes the goals of the system and its current status.
\end{abstract}

\section{Introduction}

Portfolios are widely recognized and widely used in a number of creative disciplines as a means to organize and to convey one's creative skills and output.

Guelph's NSERC Chair in Environmental Design Engineering (CEDE) required a means to track student work, student skill development and progression over time. Collecting and archiving this work by conventional means was seen as tedious, overwhelming and unsustainable. Equally conventional means were seen as serving the Chair and providing little value to faculty and students that would need to consume time and effort in support. Thus, the concept of an electronic portfolio system was seen as a means to serve the Design Chair, students and the School of Engineering.

The NSERC Chairs in Design Engineering and Chairs in Environmental Design Engineering have developed a white paper on Engineering Design Competency [1]. The document was created for engineering schools to draw from in the advancement of their programs. The white paper provides a framework with which to structure student's skills within the portfolio system.

This paper will briefly describe the desirable system features, the development process, the current system status and close with future steps.

\section{System Users and Desirable Features}

The development of the system and its specifications has been an evolutionary process. The portfolio system is intended to serve many customers. The desired features for each customer will be presented.

The student user of the system is the most important customer. The student's requirements include: easy to use without significant incremental time required to submit their work, reliable and secure, and providing added value. Added value requires that the system is appealing and effective for the other user community.

The faculty user requires a system that is: easy to use, reliable and secure, and added value. Added value includes effectively handling student's electronic work and easing administrative tasks associated with archiving work for subsequent external reviews.

The School of Engineering benefits from the system provided the system is capable of aiding promotion of the school and our students, assessment of our program quality and supporting documentation of our program and student efforts for the accreditation process.

Guests to the portfolio system include the general public, prospective students, family and friends of our students, employers and external partners. The diversity of guests leads to a range of desirable features. The appeal of the system to potential employers represents an important added value for the students.

Alumni are also seen as long term users. The portfolio system aspires to be valuable to them as students and to be valuable to them throughout their career.

\section{System Development}

The development of the system began during the summer of 2005. An initial version was subjected to initial tests during the Fall 2006 semester. This test was limited to a single course, Engineering \& Design II, taken by all engineering students at the University of Guelph.

The system was further developed following the Fall 2006 trial and "version 2" was tested in three courses during the 2007 / 2008 academic year. 


\section{System Status}

The system is currently located at folio.soe.uoguelph.ca. Figure 1 provides the introduction page.

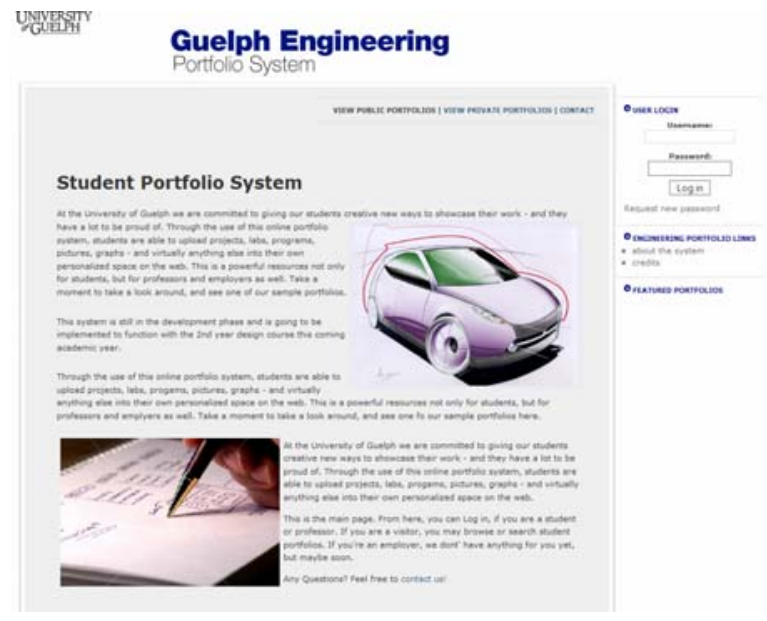

Figure 1: Portfolio System Welcome Screen

Faculty specific interface screens have been developed to permit faculty to manage their course(s) within the system. Figure 2 is one example screen. A participating faculty member can use the system in the following ways. They can manage the students in their course including identifying multiple team memberships via uploading a text file. The term projects, assignments and submissions can be entered including due dates/times and file formats. Differential due dates and times are possible for each student and/or team. The professor may track which students have submitted as a function of time. The system locks out submissions once the due date/time is passed. Faculty may also identify which skills or competencies are associated with a given submission. Finally the student's submissions can be downloaded one at a time or as a single compressed file.

A faculty member may also upload files into a student's portfolio. This feature has proved useful for uploading videotaped student presentations. It may also prove useful in uploading feedback regarding a student's submission.

A student user can upload their resume, a biographical sketch, and provide their program status. The student controls their password and the password for private viewing. For a student's participating courses, they can submit files and commentary notes for each participating assignments. Team submissions need only be submitted by one individual with this team submission automatically linked to the portfolio of all team members. A student is also able to upload files to reflect their extracurricular activities. These files might reflect summer or coop job skill development or reflect voluntary activities associated with student clubs and the like.

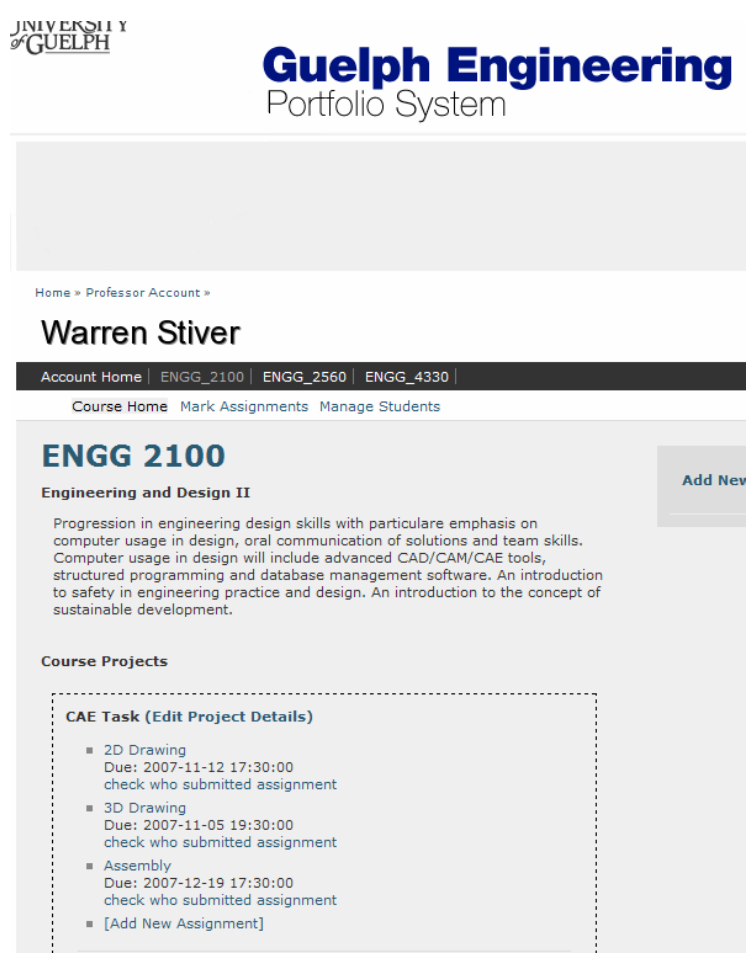

Figure 2: A Faculty User Interface

The system provides the capacity for the students to look at their portfolio from a variety of perspectives. One option is to look at their work from a skills perspective as is evident in Figure 3. Clicking on a specific skill pulls up the portfolio entries that were entered with that skill flagged. The system currently has a constrained list of skill categories but this list will grow as our usage of the system grows.

Each student controls viewing access to their portfolio. A student could choose to preclude anyone else from seeing any element of their portfolio. In this case the system supports a student's reflection on their personal skill development. Another student could choose to make everything fully publicly accessible - essentially an open website organized to reflect engineering competencies. It is expected that few students would wish to share everything with everyone. Equally it is expected that few students would wish to share none of their work with anyone. Students have generally indicated that they will be willing to share a small fraction of their work with an 
open audience, willing to share a fraction of their work with a controlled audience and content to keep a substantial portion of their work just for themselves. In this spirit, the system has been developed to allow a student to choose the level of accessibility for each and every entry. Three levels of accessibility are available - public, private and none. Public access means the work is open to all. Private access means the item is accessible to viewers that a student has provided a private viewing password. Finally none corresponds to only viewable by the student.

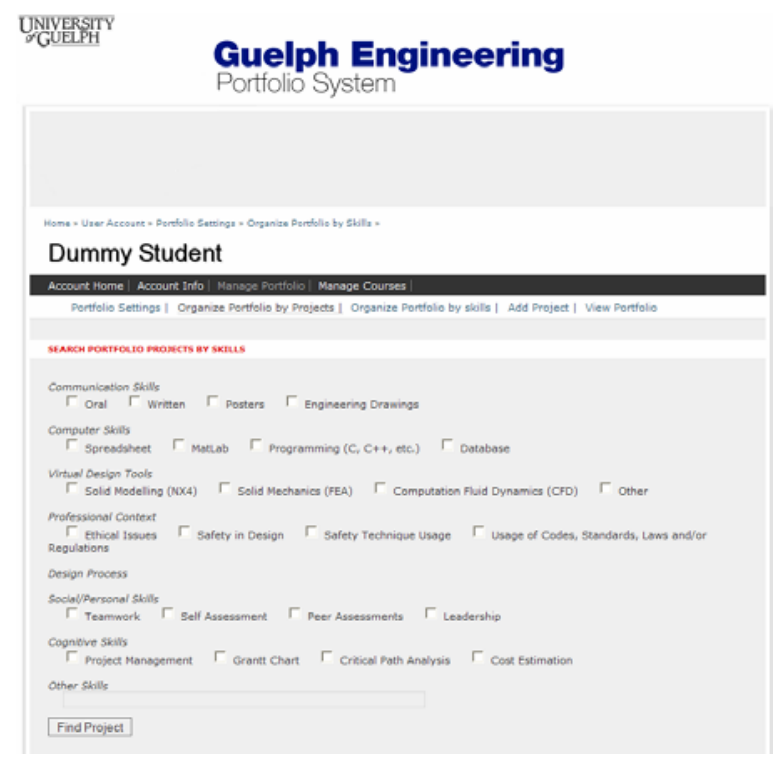

Figure 3: A Student Skills Screen

Two clarifications are important regarding viewing rules. For team projects, a public viewing depends on all members of the team choosing the public view option. All student submissions for courses are viewable by course instructors independent of the student's viewing choice.

In summary, the current status of the portfolio system is an operational system that effectively serves students and faculty in the context of submitting and archiving work in the context of individual courses. The administrative features necessary to support both student and faculty use of the system are fully functional. The system has been tested in three courses by a range of students. Feedback from students is positive but comes with many, many suggestions for expansion. Students recognize that the added value that they will realize from the system depends on the system reaching widespread usage among their courses and on the desired feature expansion.

\section{Future Plans}

A portfolio inherently reflects the collection of one's skills and output. The system's full aspirations cannot be realized in the absence of participation of the majority of a student's courses. Thus, it is a priority to increase the number of courses in the system to at least $50 \%$ within the next couple of years.

A portfolio that cannot be seen by others, particularly potential employers, loses a substantial portion of its potential value. The private view of a student's portfolio needs to be completed. The student administrative features and database features are complete for this element. The next step is the development of the most effective means to display the portfolio in the best light. In addition, navigation and search structures need to be developed. The target is to have these features fully functional by 2009 .

For faculty, Guelph's NSERC CEDE and for the School of Engineering, the portfolio system offers more than a submission system for student work. The system has the potential to help in the assessment and documentation of our progress within individual courses and most importantly within the program as a whole. It should serve to identify gaps and excessive redundancy. It should serve to demonstrate growth in our students from first to fourth year. It should serve to document outcome improvements over time in all areas including design. Serving these requirements requires building routines to automatically strip out individual student identifiers and building effective search structures. Building these features will continue over the next couple of years.

\section{References}

[1] The NSERC Chairs in Design Engineering and Environmental Design Engineering (2005). Towards a Blueprint for Educating Design Engineers: Design Competency, NSERC of Canada, 50p.

\section{Acknowledgements}

Funding for this work has been provided by NSERC through the Chairs in Environmental Design Engineering program and this funding is gratefully acknowledged. Many students have provided substantial constructive feedback regarding the system during its development stages. Their enthusiasm for the project and their feedback has been essential for its continued advancement. 